\title{
High Performance Distributed Coordination Function for Wireless LANs
}

\author{
Haithem Al-Mefleh^ and J. Morris Chang \\ Dept. of Electrical and Computer Engineering \\ Iowa State University, Ames, IA 50011, USA \\ \{almehai,morris\}@iastate.edu
}

\begin{abstract}
The performance of DCF, the basic MAC scheme in IEEE 802.11, degrades under larger network sizes, and higher loads due to higher contention and so more idle slots and higher collision rates. We propose a new high-performance DCF (HDCF) protocol that achieves a higher and more stable performance while providing fair access among all users. In HDCF, the transmitting stations randomly select the next transmitter and so active stations do not contend for the channel, and an interrupt scheme is used by newly transmitting stations without contending with the existing active stations. Thus, HDCF achieves collision avoidance and fairness without idle slots added by the backoff algorithm used in DCF. For evaluation, we provide Opnet simulation that considers saturated and non-saturated stations. Results show that HDCF outperforms DCF in terms of throughput, and long-term and short-term fairness with gains up to $391.2 \%$ of normalized throughput and $26.8 \%$ of fairness index.
\end{abstract}

\section{Introduction}

Other than being simple and distributed, the IEEE 802.11 DCF (Distributed Coordination Function) [1,2 is most popular because it assures long-term fairness (each station has the same opportunity to access the channel). In DCF, Binary-Exponential-Backoff (BEB) procedure is used to resolve collisions, and a uniform distribution is used to provide fairness property for users. A station with a packet to transmit will do so if the medium is sensed idle for a period of DIFS. Otherwise, the station sets its backoff counter by randomly choosing a number following a uniform distribution: NumberOf Backof f Slots $\sim U(0, C W)$, where $C W$ is called the contention window and is initially set to $C W_{\min }$. The station decrements its backoff counter by one for every time slot the medium is sensed idle, and transmits when this counter reaches zero. The destination responds by sending an acknowledgment (ACK) back. The packets transmitted carry the time needed to complete the transmission of a packet and its acknowledgement.

\footnotetext{
* This work is partially supported by a grant from the Information Infrastructure Institute (iCUBE) of ISU.
} 
This time is used by all other stations to defer their access to the medium and is called NAV, Network Allocation Vector. Collisions occur when two or more stations are transmitting at the same time. With every collision, the station doubles its $C W$ unless a maximum limit $C W_{\max }$ is reached, and selects a new backoff counter from the new range. The process is repeated until the packet is successfully transmitted or is dropped because a retry limit is reached. Unfortunately, such behavior degrades the performance of the network especially under higher loads due to collisions and idle slots. Even if the network has only one station transmitting, that station still has to backoff for a number of slots. In addition, collisions occur more frequently when the number of contending users increases. This results in an unstable behavior of DCF under very high loads.

In this paper, we propose a new contention management scheme named Highperformance DCF (HDCF), which addresses the problem of wasted time in contention resolution via classifying stations into active and inactive ones. Our objectives are to coordinate transmissions from different active stations with no collisions or idle slots, and limit the contention to newly transmitting stations. $\mathrm{HDCF}$ is a distributed random access scheme that achieves a higher throughput while providing long-term and short-term fairness among all users. In general, each station maintains a list of active users. The transmitting station chooses randomly the next station to transmit from its own list of active users following a uniform distribution: NextStationToTransmit $\sim U$ (first, last), where first and last are the first and last entries of the active list. The selected station transmits after a PIFS period following the last transmission, and other active stations will defer their attempts to transmit the same way NAV is used in DCF. Thus, there are no collisions or redundant idle slots due to active transmissions. On the other hand, a newly transmitting station uses an interrupt scheme. Thereafter, active stations stop their active transmissions and only new stations would contend for the channel using DCF. As a result, HDCF reduces the number of contending stations, and so collision rates, and backoff slots. Results show that HDCF outperforms DCF in terms of throughput, and fairness index with gains up to $391.2 \%$ and $26.8 \%$ respectively.

With HDCF, stations transmit in a uniform random order using a single channel with no central control, no time synchronization, no slotted channel, and no periods' reservations. In addition, HDCF utilizes an interrupt scheme so that active stations (one or more) keep transmitting unless there are new stations welling to transmit, and that those new stations (one or more) can contend directly to assure fairness preventing unbounded delays for new stations. Finally, HDCF works using the 802.11 PHY and attributes (like NAV, retry limits, fragmentation, and others), introduces no additional packets, and works with or without RTS/CTS mode (e.g. used for hidden-terminal problem).

The rest of this paper is organized as following. Related work is summarized in section 2 In section 3. HDCF protocol's details and rules are defined. A simulation study is presented in section 4 to evaluate HDCF and compare it to DCF. Finally, section 5 concludes the paper. 


\section{Related Work}

To enhance DCF, many researchers proposed schemes that mainly attempt to reduce collision rates, adapt $C W$ to congestion levels, or find optimal values of $C W$. However, collisions and wasted times still exist because some approaches solve one problem and leave another (e.g., 3,4,5]), and optimal values are approximate and oscillate with the network conditions that are variable (e.g., 3, 4, 5, 6, 10]). In addition, some schemes require the existence of an access point (AP) or complex computations (e.g., 6, 10]). Instead of providing a history of all such proposals, we will give examples that fall into these categories.

SD [3] divides $C W$ by a factor after a successful transmission to improve fairness. FCR 4 achieves a high throughput by having each station reset its $C W$ to a minimal value after a successful transmission, and double the $C W$ exponentially after a collision or losing contention. Thus, FCR requires the use of another mechanism to provide fairness. CONTI [5] attempts to fix the total number of backoff slots to a constant value. Hence, there are always idle slots and collisions may occur. In [6], the authors argued that the backoff value must be set equal to the number of stations to maximize the throughput. This algorithm requires an AP to broadcast the number of stations. Hybrid protocols (e.g. [10,11]) divide the channel into consecutive reserved contention and contention-free periods. Such protocols require a central controller, reservation, multi-channels, the use of RTS/CTS, slotted channels, and/or time synchronization. Also, new stations first wait for the contention-free periods to end resulting in unbounded delays and unfairness especially when a new station waits more than one contentionfree period. Therefore, most of these schemes limit the number of active users and lengths of different periods.

\section{$3 \quad$ HDCF Details}

HDCF utilizes an interrupt scheme and active transmissions to enhance fairness and eliminate, or reduce much of, the costs of contention of DCF (idle slots and collisions) without adding any assumptions or constraints to DCF.

\subsection{System Model, and Definitions}

The network consists of stations that share a single wireless channel. For simplicity in analysis and discussion, we assume a fully connected network; each station can listen to every other one in the network. Finally, HDCF is overlaid on 802.11 PHY layer and uses same frame formats used in 802.11 MAC.

1) Active Stations, Active-List, and Active Transmissions: active stations are those added to Active-List. Active-List contains a list of stations that have more packets to transmit, hence the name Active stations. Each station maintains its own Active-List with each entry having the format $\langle I D\rangle$ where $I D$ is the MAC address of an Active station. Active lists may not be the same in all stations. An Active transmission is started by Next-Station after a PIFS $(P I F S=S L O T+S I F S)$ following the last transmission. 
2) Next-Station: the station that is supposed to be the next transmitter and that is selected by the currently transmitting station.

3) Idle Stations, and New Stations: Idle stations are stations that have no data to transmit. New stations are those were idle, and at current time are having packets to transmit. This includes mobile stations that move into the network and have data to transmit.

\subsection{Next Station Selection, and Announcement}

Next Station Selection. The current transmitting station, the source, will randomly select an entry from its Active-List, and announce that $I D$ as NextStation by including it in the data frame. To provide fairness, a uniform distribution is used: Next-Station $=\operatorname{Uniform}(A[0], A[S i z e-1])$. Here, $A[0]$ is the first entry and $A[S i z e-1]$ is the last entry of the station's Active-List. The announcing station does not have to be active and will make an announcement even if it will not become active. This eliminates the need for active stations to contend to get back into active transmissions. Using the uniform distribution, an active station may choose itself as the next transmitter. This assures the property provided by DCF, that each station has the same opportunity to access the channel. In addition, it prevents a station from wasting any idle slots, no need to go through the backoff stages, if there are no other active stations.

Announcement. A station announces its future status by informing its neighbors that it does have or does not have more packets to transmit. In addition, a station announces Next-Station; the next station that has the right to access the channel. Using 802.11 packet formats, the "More Data" bit of the Frame Control field can be used to announce that a station is active. In addition, "Address4" of the 802.11 data frame's header can be used to announce Next-Station. This means an overhead of 6 bytes, the size of the MAC address which is small compared to the average packet size. When a station receives, or overhears, a packet with the "More Data" bit set to "1", it adds an entry to its Active-List unless that entry already exists. The entry will be $\langle I D\rangle$, where ID is the MAC address of the transmitting node. On the other hand, if the "More Data" bit is set to "0" then the entry, if exists, that has the MAC address of the transmitting node will be removed from all overhearing stations' Active-Lists.

\subsection{HDCF Rules}

When a station transmits, it also announces the Next-Station. All active stations overhearing the announcement know which station has the right to access the channel next. As shown in Fig. 1(b), Next-Station starts transmitting PIFS after the end of the last station's transmission, and SIFS is used as in DCF (Fig. 1(a) between packets of the same transmission. Also, DCF NAV is still used; stations will defer to the end of the ongoing transmission.

A new station initially follows DCF; it transmits if the channel is idle for a period of DIFS followed by backoff slots determined by Binary Exponential Backoff 


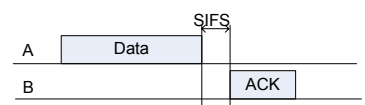

(a) An active transmission

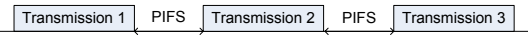

(b) Active transmissions

Fig. 1. Active transmissions and their components

as shown in Fig. 2 If there are active stations, then a new station will detect at least one active transmission since PIFS is used as the IFS between any two consecutive active transmissions and PIFS is shorter than DIFS. Thus, following DCF rules would block a new station if there are active stations. Therefore, we propose to use an interrupt scheme, Fig. 3. by which a new station uses a jam signal (the jam signal is a special signal used by many wireless MAC protocols, for instance, different jam periods are used by Black Bursts (like [7) to provide levels of priority) to stop active transmissions. If there is more than one new station interrupting, they will collide resulting in longer time spent contending for the channel. Hence, a new station starts transmitting after the jam only if the medium is idle for a period of one slot followed by backoff slots. The backoff procedure will follow the Binary Exponential Backoff procedure.

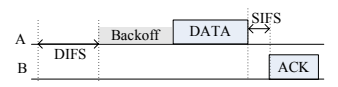

Fig. 2. Basic operation of DCF

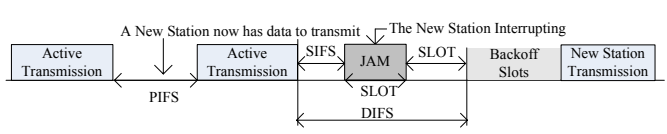

Fig. 3. The interrupt scheme

When active stations including the Next-Station detect a busy medium before the end of PIFS, as described in Fig. 3, then there is at least one new station trying to transmit. Therefore, all active stations switch back to DCF to give new stations the chance to transmit. To prevent long delays and for practical issues, active stations follow DCF after the jam signal but with EIFS (EIFS = $D I F S+S I F S+T_{A C K}$, with ACK sent using lowest PHY rate) instead of DIFS. EIFS is used only one time after the jam signal. This also provides much higher priority for new stations that use one slot after the jam. Active transmissions are reactivated by the interrupting station since it knows about at least one active station; the last announced Next-Station.

\subsection{An Example}

Fig. 4 is a simple example that illustrates HDCF operation. In the example, there are three stations that have data to transmit: $A$ with 2 packets, $B$ with 1 packet, and $C$ with 3 packets. Initially, all stations contend for the channel using DCF as they do not overhear any active transmission. Assuming that $A$ wins the contention, $A$ transmits one packet, and adds itself to its Active-List since it has another packet to transmit. The packet transmitted by $A$ will inform all 


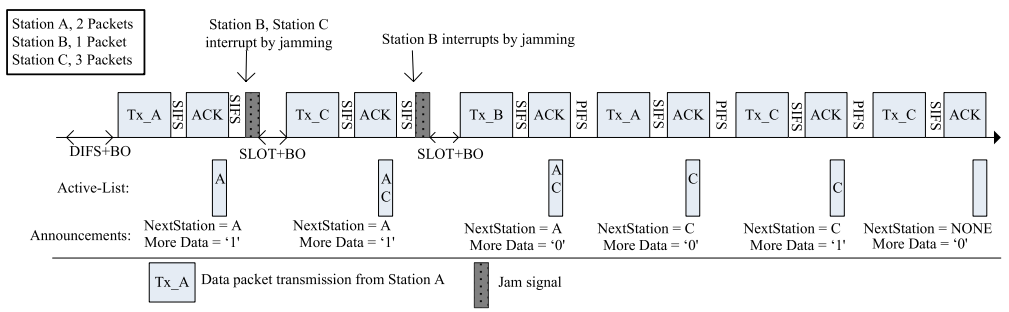

Fig. 4. An example with three stations joining network at different times

neighbors that $A$ has more packets to transmit, and announces that the next transmitter is $A$ becuase there is one entry, i.e $A$ 's MAC address, in the list and so that entry will be selected with a probability of 1 . Stations $B$ and $C$ overhears that announcement, and hence each one adds $A$ to its own Active-List.

Stations $B$ and $C$ jam for one slot SIFS after the end of the transmission of $A$. After jamming, both stations attempt to transmit after waiting for one slot followed by a random number of backoff slots. Assume that $C$ wins the contention. $C$ adds itself to its Active-List, and transmits while announcing $A$ as the next transmitter, assuming that $A$ was selected. The active list is updated at each of the three stations to include both: $A$, and $C$. Station $B$ jams the channel for one slot SIFS following the transmission of $C$, then it transmits after a period of one slot and a number of backoff slots. Note that station $B$ announces that it has no more data to transmit. Now the active list at each station includes stations $A$ and $C$. Moreover, $B$ announces $A$ as the next transmitter, and so $A$ transmits PIFS after transmission of $B$ as no more stations are interrupting. Node $A$ transmits while announcing that it has no more data and that $C$ is the next transmitter. As a result, Active-Lists at all stations are updated to include only $C$. Station $C$ transmits PIFS after the transmission of $A$ while announcing itself as the next transmitter, and that it has more data to transmit. Station $C$ transmits its last packet without any interrupt announcing no more data to transmit. All three stations update their Active-lists that become empty.

\subsection{Recovery Mechanism}

Because of hidden terminal problem, channel errors, and the sudden power-off of a station, the next selected station may not be able to start its transmission. However, all other active stations would notice the absence of Next-Stations's transmission just after a PIFS period by SIFS. Here, active stations are required to temporarily contend for the channel using DCF as a recovery mechanism, Fig. 5 shows the timings of switching to DCF (note there is no overhead to original timing in DCF since $D I F S=P I F S+S I F S)$. Also, an active station switches to DCF when it is not able to decode a packet, or the ACK is lost. Once active transmissions are recovered, the active station switches to the active state. 


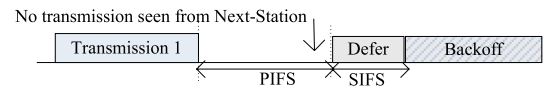

Fig. 5. Recovery from a lost Next-Station announcement

\subsection{Maximum Achieved Throughput}

The maximum saturation throughputs of a DCF and HDCF networks (ignoring the time needed by stations to join the active mode) can be approximated by:

$$
\begin{gathered}
S_{D C F}=\frac{E[L]}{D I F S+S I F S+\frac{C W_{\min } \sigma}{2}+T_{a c k}+T_{\text {data }}} \\
S_{H D C F}=\frac{E[L]}{P I F S+S I F S+T_{\text {data }}+T_{a c k}}
\end{gathered}
$$

Here, $\sigma$ is one slot time, $T_{\text {data }}$ is the time needed to send one data packet, $T_{a c k}$ is the time needed to send an ACK, and $E[L]$ is the average packet size.

\subsection{Summary, and Advantages}

An HDCF station operates in one of two modes: active mode, and contending mode. In active mode, there are no backoff, no collisions, and no idle slots. On the other hand, contending mode uses legend DCF but with much lower collision rate because almost only new stations contend for the channel. The way Next-Station is selected, and the interrupt scheme have different advantages: 1) No idle slots wasted when there are no new stations; i.e. no need to stop active transmissions. 2) Fairness to new stations as they can contend for the channel directly (like in DCF) without long delays as contention cost is much smaller. 3) Stations transmit in random order without the need for slotted channel, reserved periods, time synchronization, central control, or knowledge of number of active users. Finally, just like 802.11 DCF, HDCF stations may adapt their transmissions according to network and channel characteristics using different techniques used for the 802.11 like RTS threshold, fragmentation, link adaptation, and the use of RTS/CTS for hidden nodes.

\section{Simulation}

This section presents the simulation we used to evaluate the performance of HDCF and compare it to that of the IEEE 802.11 DCF. We utilized the commercial Opnet Modeler 11.5.A 8 to implement HDCF by modifying the 802.11 model. The simulations presented are for networks of $802.11 \mathrm{~g}$ and $802.11 \mathrm{~b}$ PHYs without RTS/CTS mode. Table 1 shows the 802.11 network parameters 2, 1, We consider different scenarios with different sizes of packets, number of stations, and offered loads to study the performance of HDCF and compare it to 
that of DCF. First, we assume a fully-connected network with no channel errors; collisions are the only source of errors. Here, we start with a saturated scenario to provide a reference and an understanding of the maximum achievable performance. Then, we study the performance under different loads, or a non-saturated network. Second, we study the performance under different noise levels. To implement noise, we used a jammer node provided by Opnet.

Table 1. Network Parameters

\begin{tabular}{|c|c|c|c|}
\hline Parameter & Value & Parameter & Value \\
\hline Slot Time & $20,20 \mu s^{1}$ & SIFS & $10,10 \mu \mathrm{s}$ \\
\hline PIFS & $30,30 \mu s$ & $D I F S$ & $50,50 \mu s$ \\
\hline$C W_{\min }$ & 15,32 & $C W_{\max }$ & 1023,1023 \\
\hline PLCP Overhead & $20,192 \mu \mathrm{s}$ & DCF MAC Overhead & 28, 28 Bytes \\
\hline$M A C A C K$ Size & 14,14 Bytes & HDCF MAC Overhead & 34,34 Bytes \\
\hline Data Rate & $54,11 M b p s$ & ControlRate & $24,1 \mathrm{Mbps}$ \\
\hline Signal Extension $^{2}$ & $6 \mu s$ & Service/Tail Size ${ }^{2}$ & 22 Bits \\
\hline
\end{tabular}

For performance analysis, we use the following metrics:

1) Normalized Throughput (Throuput/Rate MAC $_{\text {) }}$ : where throughput is the total data transmitted successfully per the simulation period.

2) Fairness Index: we used Jain Index [9] defined by $\left(J F=\frac{\left(\sum_{i=1}^{n} S_{i}\right)^{2}}{n \sum_{i=1}^{n} S_{i}^{2}}\right)$, where $n$ is number of stations and $S_{i}$ is the throughput of station $i$. The closer the value of $F I$ to 1 , the better the fairness level. We provide results for different simulation periods to have better conclusions about long and short term fairnesses.

\subsection{No Channel Noise}

Saturated Stations. Here, we compare between DCF and HDCF for a network of saturated stations, i.e stations that have data packets to transmit at all times. Fig. 6] and Fig. 7] compare the normalized throughput between HDCF and DCF for 50 contending stations as a function of the packet size, which changes from 50 bytes to 2304 bytes. The gain goes from $45.7 \%$ to $64 \%$ for $802.11 \mathrm{~b}$, and from $119.8 \%$ to $282.5 \%$ for 802.11 g. The normalized throughput increases with the packet size for both protocols. However, the gain increases as the packet size gets smaller. This is due to the fact that collision's cost is higher as it takes longer time for larger packets. In addition, the figures show the maximum normalized throughput values estimated by equations (2) for HDCF and (1) for DCF. While HDCF almost achieves the maximum throughput, DCF throughput is always much lower than the maximum possible values.

Fig. 8 compares the normalized throughput between HDCF and DCF as a function of the network size, number of stations. Here, the packet size is fixed at 1000 bytes. For 50 users, the gains are about $49.8 \%$ for $802.11 \mathrm{~b}$ and $164.7 \%$ 

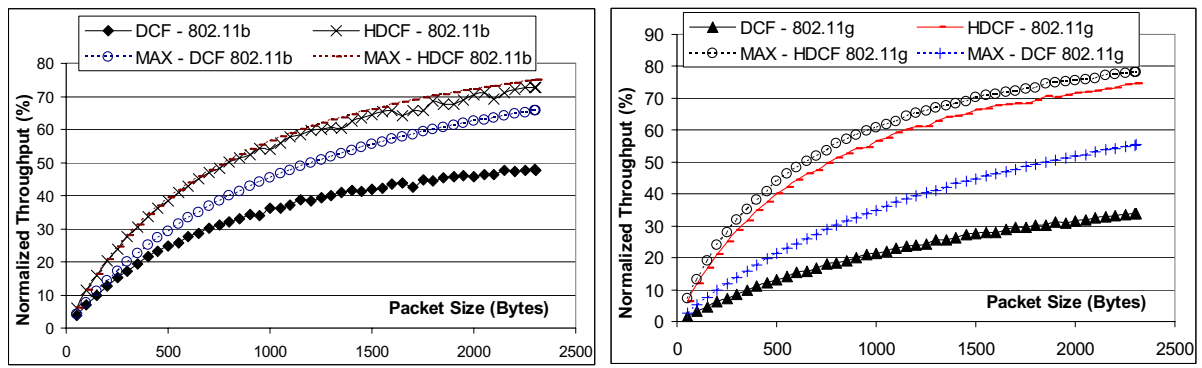

Fig. 6. Throughput vs. packet size, 802.11 b Fig. 7. Throughput vs. packet size, $802.11 \mathrm{~g}$

for $802.11 \mathrm{~g}$. Fig. 8 shows a higher stability of HDCF; the number of stations has small effect on the performance of HDCF. On the other hand, DCF performance degrades as the number of stations gets larger since the probability of collisions increases exponentially when number of stations increases. Moreover, all stations contend for the channel at all times in DCF. However, HDCF reduces the number of contending stations linearly, and remove any unnecessary idle slots. Hence, collision probability and overheads are much reduced by HDCF allowing a higher stability. In Fig. 9, we also summarize results from different simulations we conducted. The figure shows the minimum and maximum gains of normalized throughput for different network sizes. Again, packet size is changed from 50 to 2304 bytes for each network size. The least gain is $10.5 \%$, and the greatest is $391.2 \%$. In all cases, the gain increases when the number of stations increases.

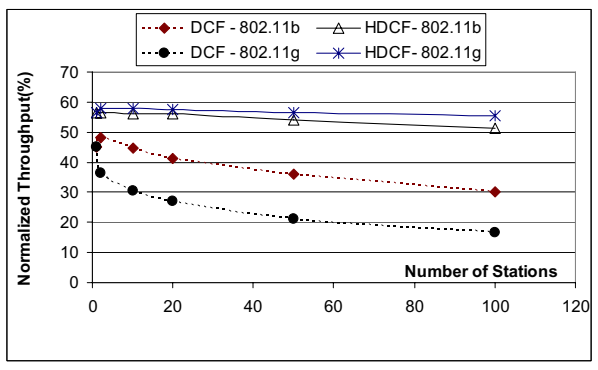

Fig. 8. Throughput vs. network size

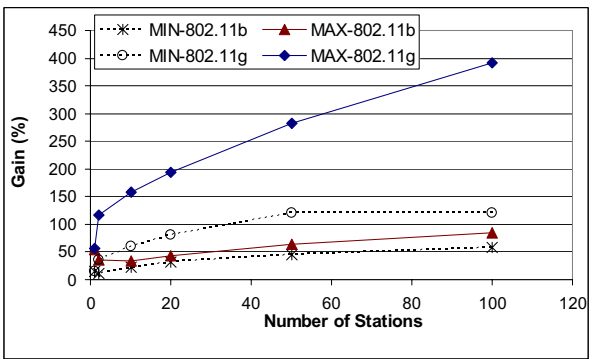

Fig. 9. Max/Min Gain

Fig. 6. Fig[7, and Fig 8 show that the higher the data rate, the lower the normalized throughput of a DCF network. In general, this is due to the higher overhead ratio. On the contrary, $\mathrm{HDCF}$ has a slightly higher performance when the rate increases because of the reduction of contention level and unnecessary idle slots which are parts of the overhead.

Fig. 10 and Fig. 11 illustrate that HDCF provides a higher short-term and long-term fairness among all stations. Here, we used an average packet size of 
1000 bytes. In HDCF, the fairness index is always above 0.84 for the 1 second simulation, and is almost 1 for the 3 seconds simulation for all sizes from 1 to 100 stations. On the other hand, the fairness index in DCF continues to decrease as the number of stations increases for both scenarios, and reaches values of 0.49 and 0.74 , respectively. For $802.11 \mathrm{~b}$, the gains are up to about $31.1 \%$ for longterm fairness, and $86.7 \%$ gain for short-term fairness. Correspondingly, the gains are up to $10.1 \%$ and $26.8 \%$ for $802.11 \mathrm{~g}$. The smaller gains in $802.11 \mathrm{~g}$ are because of the lesser time required to transmit a packet using the higher data rate.

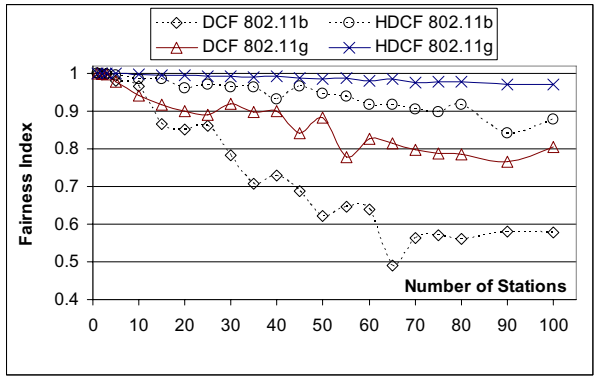

Fig. 10. Fairness Index for 1 second

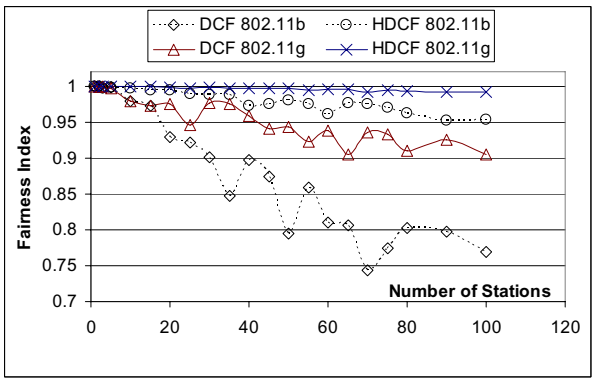

Fig. 11. Fairness Index for 3 seconds

Non-saturated stations. Here, we simulated a network of non-saturate stations to study the performance under different offered loads. We select $802.11 \mathrm{~g}$ PHY, fix number of stations in the network to 50, set packet size to 1000 bytes, and vary the offered load at every station from 1 to 400 packets per second. Each simulation is run for a 50 second period. Fig. 12 illustrates that while providing better fairness, HDCF could achieve up to $168.3 \%$ more throughput than that of DCF. From the previous experiment, we select a load rate of 20 packets per second and vary network size from 1 to 100 stations. Fig. 13 shows that HDCF concurrently improves fairness and provides throughput gains as high as $71.7 \%$.

Fig. 12 and Fig. 13 also explain that once the network load, or the network size, gets beyond a certain level, DCF performance starts to degrade due to higher contetion levels. Alternatively, HDCF enhances the performance because it reduces number of contending stations and unnecessary idle slots. Also, HDCF performs just like DCF when the network load is very light or when the network size is small where DCF is proved to be highly efficient. Thus, HDCF achieves higher performance and adapts better to different loads and network sizes.

Finally, we provide results for different simulation's periods to have better conclusions about short-term fairness. Fig. 14 presents fairness index vs. duration for a network of 80 stations, and a constant packet interarrival time of 0.01 second at each station. The gains can reach values up to $70.6 \%$ and $23.6 \%$ for $802.11 \mathrm{~b}$ and $802.11 \mathrm{~g}$ respectively. Since the gain increases for shorter periods, HDCF improves the short-term fairness of the network. Such enhancement is related to throughput's improvements as explained above, and how the next station is selected as explained in section 3.2 




Fig. 12. Throughput vs. load

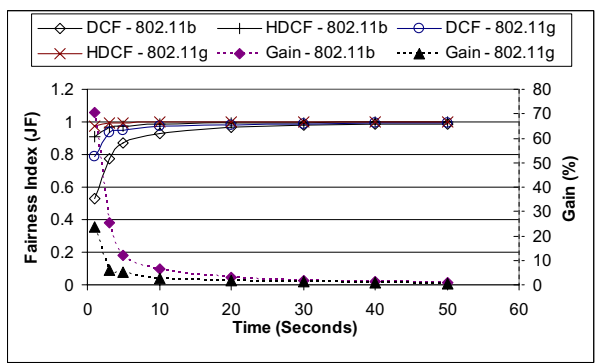

Fig. 14. Fairness Index vs. time

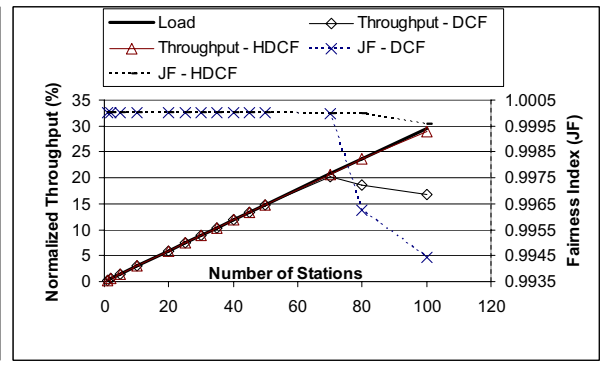

Fig. 13. Throughput vs. number of users

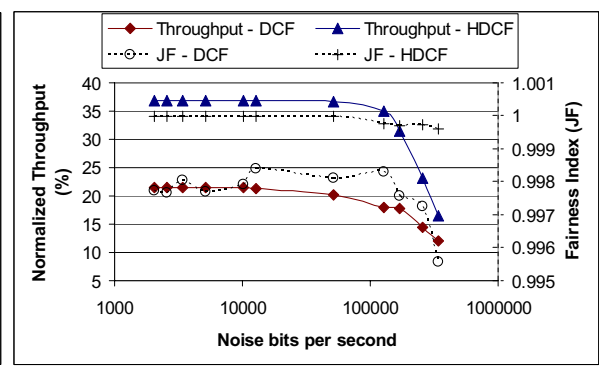

Fig. 15. Throughput vs. noise level

\subsection{Channel Noise}

The results provided are for a network of 50 stations with CBR traffic of 50 packets per second. Each packet is 1000 bytes, and each simulation is run for 100 seconds. The jammer was configured to produce noise signals with a constant length of 1024 bits/signal at a constant rate varied for different runs. Fig. [15] shows the performance measures vs. the number of noise bits per second for an 802.11g network, and has a log-scaled x-axis. The figure shows that the throughput gain increases up to about $93.7 \%$, and then starts to decrease until there is no gain when the channel errors are very high. Furthermore, HDCF provides higher throughputs and fairness for all tested noise levels.

\section{Conclusions}

In this paper, we proposed a new high-performance DCF (HDCF) MAC protocol to address the problem of wasted time in contention resolution in the IEEE 802.11 DCF. HDCF eliminates the need for unnecessary contention and idle slots by allowing transmitting stations to select the next user to transmit. To assure fairness, next station is selected in a random uniform fashion. In addition, new stations utilize an interrupt scheme to contend directly without delays. Thereafter, active stations would stop their active transmissions and only new 
stations would compete for the channel using DCF. As a result, HDCF reduces the number of contending stations, and so collision rates, and backoff slots. Also, stations transmit in a uniform random order without the need for slotted channel, reserved periods, time synchronization, central control, or knowledge of number or order of active users. We also presented a simulation study using Opnet which illustrated that HDCF significantly improves the performance as it achieves higher throughput and fairness levels for both saturation and nonsaturation scenarios. For $802.11 \mathrm{~g}$, the gains can be up to $391.2 \%$ of throughput and $26.8 \%$ of fairness index.

\section{References}

1. IEEE Std 802.11b-, Wireless LAN Medium Access Control (MAC) and Physical Layer (PHY) Specifications: Higher-Speed Physical Layer Extension in the $2.4 \mathrm{GHz}$ Band (1999)

2. IEEE Std 802.11g-, Part 11: Wireless LAN Medium Access Control (MAC) and Physical Layer (PHY) specifications Amendment 4: Further Higher Data Rate Extension in the $2.4 \mathrm{GHz}$ Band (2003)

3. Ni, Q., Aad, I., Barakat, C., Turletti, T.: Modeling and analysis of slow cw decrease for ieee 802.11 wlan (2003)

4. Kwon, Y., Fang, Y., Latchman, H.: A novel medium access control protocol with fast collision resolution for wireless lans. In: INFOCOM (2003)

5. Abichar, Z.G., Chang, J.M.: Conti: Constant-time contention resolution for wlan access. NETWORKING, 358-369 (2005)

6. Li, C., Lin, T.: Fixed Collision Rate Back-off Algorithm for Wireless Access Networks. In: VTC Fall 2002 (September 2002)

7. Sobrinho, J.L., Krishnakumar, A.S.: Real-time Traffic over the IEEE 802.11 Medium Access Control Layer. Bell Labs Technical Journal, 172-187 (1996)

8. Opnet, Opnet Modeler, http://www.opnet.com

9. Jain, D.R.: Chiu, and W. Hawe, A Quantitative Measure of Fairness and Discrimination for Resource Allocation in Shared Computer Systems, DEC Research Report TR-301 (September 1984)

10. Alonso, L., Ferrus, R., Agusti, R.: Wlan throughput improvement via distributed queuing mac. Communications Letters. IEEE 9(4), 310-312 (2005)

11. Chlamtac, I., Farago, A., Myers, A., Syrotiuk, V., Zaruba, G.: Adapt: a dynamically self-adjusting media access control protocol for ad hoc-networks. In: Global Telecommunications Conference. GLOBECOM 1999, vol. 1A(1a), pp. 11-15 (1999) 